\begin{tabular}{l|l|l|}
\cline { 2 - 3 } CRITICAL & $\begin{array}{l}\text { Western Ghats } \\
\text { PARTNERSHIP FUND }\end{array}$ & Special Series \\
\hline
\end{tabular}

\title{
Birds of lower Palni Hills, Western Ghats, Tamil Nadu
}

\author{
Tharmalingam Ramesh ${ }^{1}$, J. Peter Prem Chakravarthi ${ }^{2}$, S. Balachandran ${ }^{3}$ \& Riddhika Kalle ${ }^{4}$ \\ 1,4 Wildlife Institute of India, Chandrabani, P.O. Box 18, Dehra Dun, Uttarakhand 248001, India \\ 1,2,3 Bombay Natural History Society, Hornbill House, Shaheed Bhagat Singh Road, Mumbai, Maharashtra 400001, India \\ ${ }^{4}$ B-1, Prerana Mandir, S.V. Road, Santacruz(W), Mumbai, Maharashtra 400054, India \\ Email: ${ }^{1}$ ramesh81ngl@gmail.com (corresponding author), ${ }^{2}$ peterpremcj@gmail.com, ${ }^{3}$ bnhsbala@rediffmail.com, ${ }^{4}$ riddhikalle@gmail.com
}

Date of publication (online): 26 November 2012 Date of publication (print): 26 November 2012 ISSN 0974-7907 (online) | 0974-7893 (print)

Editor: Kumaran Sathasivam

\section{Manuscript details:}

Ms \# 03051

Received 02 January 2012

Final received 14 May 2012

Finally accepted 10 October 2012

Citation: Ramesh, T., J.P.P. Chakravarthi, S. Balachandran \& R. Kalle (2012). Birds of lower Palni Hills, Western Ghats, Tamil Nadu. Journal of Threatened Taxa 4(14): 3269-3283.

Copyright: (c) Tharmalingam Ramesh, J. Peter Prem Chakravarthi, S. Balachandran \& Riddhika Kalle 2012 Creative Commons Attribution 3.0 Unported License. JoTT allows unrestricted use of this article in any medium for non-profit purposes, reproduction and distribution by providing adequate credit to the authors and the source of publication.

Author Details and Author Contribution: see end of this article.

Acknowledgements: The Ministry of Environment and Forest, Government of India is greatly acknowledged for funding our research activities in the Palni Hills. We thank the Director, Bombay Natural History Society and Tamil Nadu Forest Department for granting us the permission to carry out our study in the Palni Hills. We are indebted to our field assistants and forest department staff for their help and logistic support.
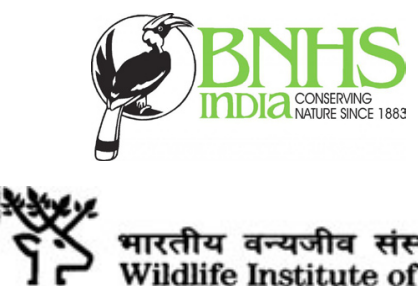

भारतीय वन्यजीव संस्थान Wildlife Institute of India

urn:Isid:zoobank.org:pub:04D8DDC9-27974E38-BA36-72C91C3F94EE

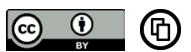

OPEN ACCESS | FREE DOWNLOAD
Abstract: The current altitudinal distribution and breeding observations on birds of lower Palni Hills, Western Ghats were documented by conducting road transects, opportunistic surveys including trail walks and mist netting. A total of 196 species belonging to 63 families were recorded during the study. The Accipitridae family was foremost in species richness, followed by Cuculidae and Muscicapidae, Picidae, Timaliidae and other families. Altitudinal distribution of birds was higher between 600 and $900 \mathrm{~m}$. The general patterns of the decreasing species richness with increasing altitude were observed in mid and upper Palnis. This could be probably because the lower Palnis have more deciduous and scrub forest which can support high food availability. Resident and migrant species made up to $87.76 \%$ and $12.24 \%$ of the community, respectively. We recorded a species that was threatened, three nearly threatened, and five endemic to the Western Ghats. Most of the endemics were confined to the higher altitudes due to the presence of moist evergreen and high altitude montane forests and grasslands. In total, 51 breeding bird observations were recorded. Interestingly, the variation in the breeding season of some birds was noticed with respect to earlier studies. Overall, our study illustrated useful information on bird community in this region which serves as a baseline for future monitoring programs.

Keywords: Altitudinal distribution, breeding birds, lower Palni Hills, Western Ghats.

\section{INTRODUCTION}

Birds are ideal bioindicators and useful models for studying a variety of environmental problems, hence the condition of local landscape must be investigated to identify crucial determinants of the bird community structure for avian conservation (Kattan \& Franco 2004). Describing and explaining spatial patterns in species diversity are crucial steps in conserving global biodiversity (Lee et al. 2004) as the number of bird species inhabiting various altitudinal belts or 'life zones' (Ali 1949) depend on climatic changes accompanied by corresponding changes in vegetation. Studies on species distribution along elevational gradients are essential to understand principles of community organisation and species conservation. The Western Ghats, identified as one of the biodiversity hotspots in the world, is a $1600-\mathrm{km}$ long chain of mountain ranges running parallel to the western coast of the Indian peninsula (Pascal 1988). Among the three distinct sections of the Western Ghats, the south-

The publication of this article is supported by the Critical Ecosystem Partnership Fund (CEPF) -- a joint initiative of l'Agence Française de Développement, Conservation International, the Global Environment Facility, the Government of Japan, the MacArthur Foundation and the World Bank 
eastern region has one of the richest tropical forests in the country. The Western Ghats has nearly 508 bird species of which $3.5 \%$ are endemic out of more than 1200 species in India (Rajmohana \& Radhakrishnan 2008). Apparently the Indian bird population has been dwindling due to direct/indirect impact from increasing human population (Balachandran et al. 2005), habitat loss, fragmentation, and severe biotic pressure, necessitating documentation of the current status of bird species for future monitoring and conservation (Islam \& Rahmani 2004). Bird community evaluation has become an important tool in biodiversity conservation and for identifying conservation actions in areas of high human pressure (Kremen 1992; Shafiq et al. 1997; Chettri et al. 2001; Ramesh et al. 2011).

The avifaunal diversity of Palni Hills has been documented by various authors (Nichols 1937; Fairbank 1877; Terry 1887) and specific ecological studies were carried out in the higher altitudes (Balachandran 1998; Robin \& Sukumar 2002; Balachandran et al. 2005; Somasundaram \& Vijayan 2008). Apart from anecdotal observations on birds (Blanford 1867; Foulkes 1904; Navarro 1955, Fuller 1958; Surendran 1973; Ananthasubramaniam 1979; Narayana 1979; Steele 1990), few studies involve bird ringing techniques (Balachandran 1992) and a few have been monitored at low altitudes (Shahabuddin 1997). However, little is known about the avifaunal status in the lower Palni Hills, from the foothills to an altitude of $900 \mathrm{~m}$ above mean sea level. In order to fill up the lacunae, a short term survey was carried out to document the avifaunal altitudinal distribution and breeding observations in the lower Palni Hills.

\section{MATERIALS AND METHODS}

\section{Study Area}

Palni Hills is in the south-eastern part of the Western Ghats Hotspot in Tamil Nadu. It lies between $10.1^{0}-10.26^{\circ} \mathrm{N}$ and $77.14^{0}-77.52^{\circ} \mathrm{E}$ covering an area of c. $2400 \mathrm{~km}^{2}$ (Image 1). It is one of the important global biodiversity hotspots with high endemism. The Palni Hills have three well marked topographic divisions, namely the lower (300-900 m), mid ( $>900$ $1800 \mathrm{~m}$ ) and high Palnis ( $>1800-2500 \mathrm{~m})$ for research and administrative purposes (Balachandran et al. 2005). Perennial rivers such as Mulayar and Arunganal and semiperennial rivers like Thalaiyar (Rat-tail Falls) (Balachandran et al. 2005) and Iruttar, are important water sources for the Manjalaar reservoir (situated at the foothills of $300 \mathrm{~m}$ elevation) and it is used for irrigation purposes in the lower Palnis. The minimum recorded temperature is about $8.3^{\circ} \mathrm{C}$ and the maximum goes up to $23^{\circ} \mathrm{C}$. The annual average precipitation ranges from $800-1900 \mathrm{~mm}$ in the Palnis (Balachandran et al.

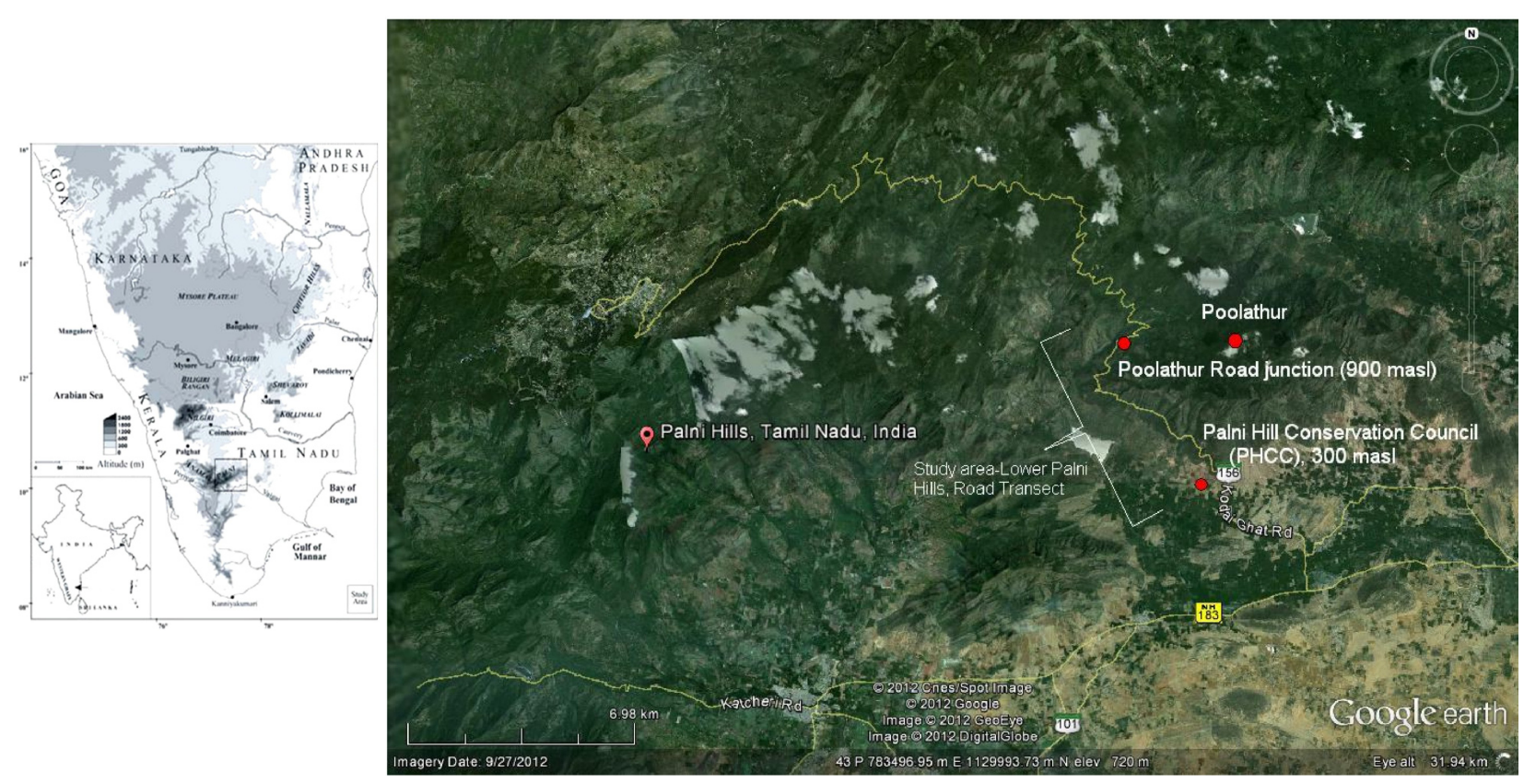

Image 1. Location of intensive study area in lower Palni Hills, Western Ghats. 
2005). There are four seasons; Winter (DecemberMarch), Summer (April-June), southwest monsoon (June-September) and northeast monsoon (OctoberNovember). The climate is sub-tropical with major vegetation types broadly classified into scrub forest, moist and dry deciduous forest of low and mid elevations and montane evergreen forest of high elevations (Champion \& Seth 1968). In addition to birds, important mammals found in Palni are Sambar Rusa unicolor, Muntjac Muntiacus muntjak, Wild Pig Sus scrofa, Indian Chevrotain Tragulus meminna, Gaur Bos gaurus, Four-horned Antelope Tertracerus quadricornis, Nilgiri Tahr Nilgiritragus hylocrius, Tiger Panthera tigris, Leopard Panthera pardus, Dhole Cuon alpinus, Striped Hyena Hyaena hyaena, Golden Jackal Canis aureus and Grizzled Giant Squirrel Ratufa macroura (Prater 1971).

The Manjalaar reservoir forms an important water body for water birds. Some parts of the river delta in lower Palnis constituted an endemic eco-region with very rare and endangered plants and animals of India but now it has been modified with cultivation of Silk Cotton Ceiba pentandra, Teak Tectona grandis, Mango Mangifera indica, Coffee Coffea arabica and Coconut Cocos nucifera. Expanding agricultural, urban, productive and developmental activities currently threaten to take over Palni's natural habitat and pose a threat to its preservation due to large-scale deforestation. With human disturbance, the riparian forest patch is being converted on the slopes as a complex mosaic of plantations such as mango groves, groundnut and pulses, secondary and native forests. This work is part of a long term research project "Revaluation of bird community structure of Palni Hills with special reference to endemic and threatened species" (Balachandran et al. 2005).

\section{Field survey}

We recorded sightings of birds in the lower Palnis from December 2004 to July 2005 and documented the occurrence, altitudinal distribution and breeding. The highly mountainous terrain made it impossible to lay line transects. Instead, we conducted road transects in the lower Palnis from Palni Hill Conservation Council (PHCC), Kamakapatti to Poolathur road junction $(16.5 \mathrm{~km})$. The road was divided into six segments of $2.5 \mathrm{~km}$ each, including a segment of $4 \mathrm{~km}$ where each segment was sampled twice every month in the mornings (06:00-10:00 hr) and evenings (16:00-18:30 hr). In addition, opportunistic road surveys, trail walks and mist netting were conducted to record bird species within a $3 \mathrm{~km}$ radius from the regular road transect area. The five mist-nets were operated opportunistically at dawn from 06:00-09:00 $\mathrm{hr}$ and in the evenings from 15:00-18:00 hr. Mist nets were visited every hour to check the trapped birds for collection and identification. All nets were made of black nylon mesh size, $36 \mathrm{~mm}$ with dimensions $2 \mathrm{~m}$ high by $6 \mathrm{~m}$ long.

\section{RESULTS}

A total of 196 species belonging to 63 families were recorded during the study (Table 1). All observations listed in Table 1 have already been published in the survey report (Balachandran et al. 2005). Accipitridae family showed highest species richness (18 species), followed by Cuculidae and Muscicapidae (10 species each), Picidae, Timaliidae, (eight species each), Phasianidae and Pycnonotidae (seven species each), Ardeidae, Cisticolidae, Columbidae, Dicruridae, Estrildidae, Motacillidae and Nectariniidae (five species each) and other families (less than five species each). Of the recorded species, $87.76 \%$ were resident and $12.24 \%$ migrants. In terms of frequency of observations, the majority of species were found to be common (74.49\%), followed by uncommon (19.90\%) and rare $(5.61 \%)$ species. In terms of altitude, species and families were seen between $>600-\leq 700 \mathrm{~m}$ and $>700-900 \mathrm{~m}$ respectively. The number of bird species observed in each altitude zone were $128-\leq 400 \mathrm{~m}$, $122->400$ to $\leq 500,121->500$ to $\leq 600,139->600$ to $\leq 700,131->700$ to $\leq 800$ and $130->800$ to $\leq 900$. During the study, a threatened species, three nearly threatened and 192 least concerned species were also recorded. Among all the species, five were endemic to the Western Ghats.

\section{Breeding observations}

Breeding observations of 51 bird species are given as follows:

Shikra Accipiter badius: In the lower Palnis, (c. $350 \mathrm{~m}$ altitude) a mating pair was sighted on 3 February 2005. On 25 February, its nest was noticed with an egg on an Ailanthus excelsa tree and was seen with two 
Table 1. List of birds observed in lower Palni Hills, Western Ghats

\begin{tabular}{|c|c|c|c|c|c|}
\hline & Family/Species common name & Scientific name & Status & $\begin{array}{l}\text { Altitude } \\
\text { (in m) }\end{array}$ & $\begin{array}{c}\text { Frequency } \\
\text { of observation }\end{array}$ \\
\hline & Accipitridae & & & & \\
\hline 1 & Black-shouldered Kite & Elanus caeruleus & $\mathrm{R}$ & $320-900$ & C \\
\hline 2 & Oriental Honey-Buzzard & Pernis ptilorhynchus & $\mathrm{R}$ & $600-900$ & UC \\
\hline 3 & Black Kite & Milvus migrans & $\mathrm{R}$ & $300-900$ & C \\
\hline 4 & Brahminy Kite & Haliastur indus & $\mathrm{R}$ & $300-900$ & C \\
\hline 5 & Shikra & Accipiter badius & $\mathrm{R}$ & $300-900$ & C \\
\hline 6 & Crested Goshawk & Accipiter trivirgatus & $\mathrm{R}$ & $300-900$ & C \\
\hline 7 & Besra Sparrowhawk & Accipiter virgatus & $\mathrm{R}$ & 650 & C \\
\hline 8 & White-eyed Buzzard & Butastur teesa & $\mathrm{R}$ & 320 & $\mathrm{C}$ \\
\hline 9 & Mountain Hawk-Eagle & Spizaetus nipalensis & $\mathrm{R}$ & $415-900$ & UC \\
\hline 10 & Changeable Hawk-Eagle & Spizaetus cirrhatus & $\mathrm{R}$ & $300-900$ & C \\
\hline 11 & Bonelli's Hawk-Eagle & Hieraaetus fasciatus & $\mathrm{R}$ & $300-600$ & C \\
\hline 12 & Booted Eagle & Hieraaetus pennatus & M & $300-600$ & UC \\
\hline 13 & Black Eagle & Ictinaetus malayensis & $\mathrm{R}$ & $300-900$ & C \\
\hline 14 & Pallid Harrier & Circus macrourus & $\mathrm{M}, \mathrm{NT}$ & 320 & $\mathrm{R}$ \\
\hline 15 & Montagu's Harrier & Circus pygargus & $M$ & 350 & $\mathrm{R}$ \\
\hline 16 & Pied Harrier & Circus melanoleucos & M & $800-900$ & UC \\
\hline 17 & Short-toed Snake-Eagle & Circaetus gallicus & $\mathrm{R}$ & $300-600$ & UC \\
\hline \multirow[t]{2}{*}{18} & Crested Serpent Eagle & Spilornis cheela & $\mathrm{R}$ & $400-900$ & C \\
\hline & Acrocephalidae & & & & \\
\hline 19 & Thick-billed Warbler & Acrocephalus aedon & M & $320-500$ & UC \\
\hline 20 & Blyth's Reed-Warbler & Acrocephalus dumetorum & M & $300-900$ & $\mathrm{C}$ \\
\hline \multirow[t]{2}{*}{21} & Paddyfield Warbler & Acrocephalus agricola & M & $300-500$ & C \\
\hline & Aegithinidae & & & & \\
\hline \multirow[t]{2}{*}{22} & Common lora & Aegithina tiphia & $\mathrm{R}$ & $300-900$ & C \\
\hline & Alaudidae & & & & \\
\hline 23 & Jerdon's Bush-Lark & Mirafra affinis & $\mathrm{R}$ & 320 & C \\
\hline 24 & Ashy-crowned Sparrow-Lark & Eremopterix grisea & $\mathrm{R}$ & 320 & UC \\
\hline \multirow[t]{2}{*}{25} & Eastern Skylark & Alauda gulgula & $\mathrm{R}$ & 320 & UC \\
\hline & Alcedinidae & & & & \\
\hline \multirow[t]{2}{*}{26} & Small Blue Kingfisher & Alcedo atthis & $\mathrm{R}$ & $320-900$ & C \\
\hline & Anatidae & & & & \\
\hline 27 & Northern Pintail & Anas acuta & M & 300 & UC \\
\hline \multirow[t]{2}{*}{28} & Spot-billed Duck & Anas poecilorhyncha & $\mathrm{R}$ & 300 & $\mathrm{C}$ \\
\hline & Apodidae & & & & \\
\hline 29 & Indian Edible-nest Swiftlet & Collocalia unicolor & $\mathrm{R}$ & $600-900$ & C \\
\hline 30 & White-rumped Needletail & Zoonavena sylvatica & $\mathrm{R}$ & 630 & UC \\
\hline 31 & House Swift & Apus affinis & $\mathrm{R}$ & $320-640$ & C \\
\hline \multirow[t]{2}{*}{32} & Asian Palm-Swift & Cypsiurus balasiensis & $\mathrm{R}$ & $300-500$ & UC \\
\hline & Ardeidae & & & & \\
\hline 33 & Indian Pond-Heron & Ardeola grayii & $\mathrm{R}$ & $300-900$ & $\mathrm{C}$ \\
\hline 34 & Cattle Egret & Bubulcus ibis & $\mathrm{R}$ & 300 & C \\
\hline 35 & Median Egret & Mesophoyx intermedia & $\mathrm{R}$ & 300 & C \\
\hline 36 & Large Egret & Casmerodius albus & $\mathrm{R}$ & 300 & UC \\
\hline
\end{tabular}




\begin{tabular}{|c|c|c|c|c|c|}
\hline & Family/Species common name & Scientific name & Status & $\begin{array}{l}\text { Altitude } \\
\text { (in m) }\end{array}$ & $\begin{array}{c}\text { Frequency } \\
\text { of observation }\end{array}$ \\
\hline \multirow[t]{2}{*}{37} & Little Egret & Egretta garzetta & $\mathrm{R}$ & 300 & $\mathrm{C}$ \\
\hline & Bucerotidae & & & & \\
\hline \multirow[t]{2}{*}{38} & Malabar Grey Hornbill & Ocyceros griseus & $\mathrm{R}, \mathrm{E}$ & $800-900$ & C \\
\hline & Campephagidae & & & & \\
\hline 39 & Pied Flycatcher-Shrike & Hemipus picatus & $\mathrm{R}$ & $600-900$ & $\mathrm{C}$ \\
\hline 40 & Black-headed Cuckoo-Shrike & Coracina melanoptera & $\mathrm{R}$ & $300-900$ & UC \\
\hline 41 & Scarlet Minivet & Pericrocotus flammeus & $\mathrm{R}$ & $500-900$ & C \\
\hline \multirow[t]{2}{*}{42} & Small Minivet & $\begin{array}{l}\text { Pericrocotus } \\
\text { cinnamomeus }\end{array}$ & $\mathrm{R}$ & $320-680$ & C \\
\hline & Caprimulgidae & & & & \\
\hline 43 & Jerdon's Nightjar & Caprimulgus atripennis & $\mathrm{R}$ & $450-850$ & UC \\
\hline \multirow[t]{2}{*}{44} & Common Indian Nightjar & Caprimulgus asiaticus & $\mathrm{R}$ & $320-900$ & $\mathrm{C}$ \\
\hline & Cerylidae & & & & \\
\hline \multirow[t]{2}{*}{45} & Lesser Pied Kingfisher & Ceryle rudis & $\mathrm{R}$ & $300-900$ & C \\
\hline & Charadriidae & & & & \\
\hline 46 & Red-wattled Lapwing & Vanellus indicus & $\mathrm{R}$ & $300-900$ & C \\
\hline \multirow[t]{2}{*}{47} & Little Ringed Plover & Charadrius dubius & $\mathrm{R}$ & 300 & C \\
\hline & Chloropseidae & & & & \\
\hline 48 & Gold-fronted Chloropsis & Chloropsis aurifrons & $\mathrm{R}$ & $450-900$ & C \\
\hline \multirow[t]{2}{*}{49} & Jerdon's Chloropsis & $\begin{array}{l}\text { Chloropsis } \\
\text { cochinchinensis }\end{array}$ & $\mathrm{R}$ & $500-640$ & UC \\
\hline & Ciconiidae & & & & \\
\hline \multirow[t]{2}{*}{50} & Painted Stork & Mycteria leucocephala & $\mathrm{R}, \mathrm{NT}$ & 300 & UC \\
\hline & Cinclosomatidae & & & & \\
\hline \multirow[t]{2}{*}{51} & Spotted Babbler & Pellorneum ruficefs & $\mathrm{R}$ & $415-900$ & C \\
\hline & Cisticolidae & & & & \\
\hline 52 & Franklin's Prinia & Prinia hodgsonii & $\mathrm{R}$ & $550-720$ & UC \\
\hline 53 & Plain Prinia & Prinia inornata & $\mathrm{R}$ & $320-900$ & C \\
\hline 54 & Ashy Prinia & Prinia socialis & $\mathrm{R}$ & $500-900$ & $\mathrm{C}$ \\
\hline 55 & Jungle Prinia & Prinia sylvatica & $\mathrm{R}$ & $300-900$ & $\mathrm{C}$ \\
\hline \multirow[t]{2}{*}{56} & Common Tailor Bird & Orthotomus sutorius & $\mathrm{R}$ & $300-900$ & C \\
\hline & Columbidae & & & & \\
\hline 57 & Blue Rock Pigeon & Columba livia & $\mathrm{R}$ & $300-900$ & $\mathrm{C}$ \\
\hline 58 & Eurasian Collared Dove & Streptopelia decaocto & $\mathrm{R}$ & $320-400$ & UC \\
\hline 59 & Spotted Dove & Streptopelia chinensis & $\mathrm{R}$ & $300-900$ & $\mathrm{C}$ \\
\hline 60 & Little Brown Dove & Streptopelia senegalensis & $\mathrm{R}$ & $300-400$ & C \\
\hline \multirow[t]{2}{*}{61} & Emerald Dove & Chalcophaps indica & $\mathrm{R}$ & $500-900$ & C \\
\hline & Coraciidae & & & & \\
\hline \multirow[t]{2}{*}{62} & Indian Roller & Coracias benghalensis & $\mathrm{R}$ & $300-350$ & C \\
\hline & Corvidae & & & & \\
\hline 63 & Indian Treepie & Dendrocitta vagabunda & $\mathrm{R}$ & $300-900$ & $\mathrm{C}$ \\
\hline 64 & House Crow & Corvus splendens & $\mathrm{R}$ & $300-900$ & C \\
\hline \multirow[t]{2}{*}{65} & Jungle Crow & Corvus macrorhynchos & $\mathrm{R}$ & $300-900$ & $\mathrm{C}$ \\
\hline & Cuculidae & & & & \\
\hline 66 & Pied Crested Cuckoo & Clamator jacobinus & $\mathrm{R}$ & $300-450$ & $\mathrm{C}$ \\
\hline 67 & Brainfever Bird & Hierococcyx varius & $\mathrm{R}$ & $300-900$ & C \\
\hline
\end{tabular}




\begin{tabular}{|c|c|c|c|c|c|}
\hline & Family/Species common name & Scientific name & Status & $\begin{array}{l}\text { Altitude } \\
\text { (in } \mathrm{m} \text { ) }\end{array}$ & $\begin{array}{c}\text { Frequency } \\
\text { of observation }\end{array}$ \\
\hline 68 & Indian Cuckoo & Cuculus micropterus & $\mathrm{R}$ & 640 & $\mathrm{C}$ \\
\hline 69 & Indian Plaintive Cuckoo & Cocamantis passerinus & $\mathrm{R}$ & 320 & $\mathrm{C}$ \\
\hline 70 & Drongo Cuckoo & Surniculus lugubris & $\mathrm{R}$ & $300-900$ & UC \\
\hline 71 & Asian Koel & Eudynamys scolopacea & $\mathrm{R}$ & $300-900$ & C \\
\hline 72 & Small Green-billed Malkoha & $\begin{array}{l}\text { Phaenicophaeus } \\
\text { viridirostris }\end{array}$ & $\mathrm{R}$ & $300-900$ & $\mathrm{C}$ \\
\hline 73 & Sirkeer Malkoha & $\begin{array}{l}\text { Phaenicophaeus } \\
\text { leschenaultii }\end{array}$ & $\mathrm{R}$ & $300-900$ & $\mathrm{C}$ \\
\hline 74 & Greater Coucal & Centropus sinensis & $\mathrm{R}$ & $300-900$ & $\mathrm{C}$ \\
\hline \multirow[t]{2}{*}{75} & Lesser Coucal & Centropus bengalensis & $\mathrm{R}$ & $300-900$ & $\mathrm{C}$ \\
\hline & Dicaeidae & & & & \\
\hline 76 & Thick-billed Flowerpecker & Dicaeum agile & $\mathrm{R}$ & $400-600$ & $\mathrm{C}$ \\
\hline 77 & Tickell's Flowerpecker & Dicaeum erythrorhynchos & $\mathrm{R}$ & $300-600$ & C \\
\hline \multirow[t]{2}{*}{78} & Plain Flowerpecker & Dicaeum concolor & $\mathrm{R}$ & $800-900$ & C \\
\hline & Dicruridae & & & & \\
\hline 79 & Black Drongo & Dicrurus macrocercus & $\mathrm{R}$ & $300-500$ & $\mathrm{C}$ \\
\hline 80 & Ashy Drongo & Dicrurus leucophaeus & M & $450-500$ & $\mathrm{C}$ \\
\hline 81 & White-bellied Drongo & Dicrurus caerulescens & $\mathrm{R}$ & $320-700$ & $\mathrm{C}$ \\
\hline 82 & Bronzed Drongo & Dicrurus aeneus & $\mathrm{R}$ & $900-1300$ & UC \\
\hline \multirow[t]{2}{*}{83} & Greater Racket-tailed Drongo & Dicrurus paradiseus & $\mathrm{R}$ & $750-1100$ & C \\
\hline & Estrildidae & & & & \\
\hline 84 & White-throated Munia & Lonchura malabarica & $\mathrm{R}$ & $300-600$ & $\mathrm{C}$ \\
\hline 85 & White-rumped Munia & Lonchura striata & $\mathrm{R}$ & $300-900$ & $\mathrm{C}$ \\
\hline 86 & Black-throated Munia & Lonchura kelaarti & $\mathrm{R}$ & $650-900$ & UC \\
\hline 87 & Spotted Munia & Lonchura punctulata & $\mathrm{R}$ & $300-900$ & C \\
\hline \multirow[t]{2}{*}{88} & Black-headed Munia & Lonchura malacca & $\mathrm{R}$ & $300-600$ & C \\
\hline & Falconidae & & & & \\
\hline \multirow[t]{2}{*}{89} & Common Kestral & Falco tinnunculus & $\mathrm{R}$ & $300-900$ & C \\
\hline & Halcyonidae & & & & \\
\hline 90 & Stork-billed Kingfisher & Halcyon capensis & $\mathrm{R}$ & 300 & UC \\
\hline \multirow[t]{2}{*}{91} & White-breasted Kingfisher & Halcyon smrynensis & $\mathrm{R}$ & $300-900$ & C \\
\hline & Hemiprocnidae & & & & \\
\hline \multirow[t]{2}{*}{92} & Crested Tree-Swift & Hemiprocne coronata & $\mathrm{R}$ & $300-900$ & $\mathrm{C}$ \\
\hline & Hirundinidae & & & & \\
\hline 93 & Dusky Crag Martin & Hirundo concolor & $\mathrm{R}$ & $800-900$ & $\mathrm{C}$ \\
\hline 94 & Common Swallow & Hirundo rustica & M & $300-600$ & C \\
\hline \multirow[t]{2}{*}{95} & Red-rumped Swallow & Hirundo daurica & $\mathrm{R}$ & $320-900$ & C \\
\hline & Irenidae & & & & \\
\hline \multirow[t]{2}{*}{96} & Asian Fairy Bluebird & Irena puella & $\mathrm{R}$ & $600-900$ & $\mathrm{C}$ \\
\hline & Laniidae & & & & \\
\hline 97 & Bay-backed Shrike & Lanius vittatus & $\mathrm{R}$ & 320 & C \\
\hline 98 & Rufous-backed Shrike & Lanius schach & $\mathrm{R}$ & $300-900$ & $\mathrm{C}$ \\
\hline \multirow[t]{2}{*}{99} & Brown Shrike & Lanius cristatus & M & $300-900$ & $\mathrm{C}$ \\
\hline & Megalaimidae & & & & \\
\hline 100 & Brown-headed Barbet & Megalaima zeylanica & $\mathrm{R}$ & $400-850$ & C \\
\hline 101 & White-cheeked Barbet & Megalaima viridis & $\mathrm{R}$ & $700-900$ & C \\
\hline
\end{tabular}




\begin{tabular}{|c|c|c|c|c|c|}
\hline & Family/Species common name & Scientific name & Status & $\begin{array}{l}\text { Altitude } \\
\text { (in m) }\end{array}$ & $\begin{array}{c}\text { Frequency } \\
\text { of observation }\end{array}$ \\
\hline 102 & Crimson-throated Barbet & Megalaima rubricapilla & $\mathrm{R}$ & $700-900$ & $\mathrm{C}$ \\
\hline \multirow[t]{2}{*}{103} & Coppersmith Barbet & $\begin{array}{l}\text { Megalaima } \\
\text { haemacephala }\end{array}$ & $\mathrm{R}$ & $400-900$ & $\mathrm{C}$ \\
\hline & Meropidae & & & & \\
\hline 104 & Chestnut-headed Bee-eater & Merops leschenaulti & $\mathrm{R}$ & $400-900$ & C \\
\hline 105 & Small Bee-eater & Merops orientalis & $\mathrm{R}$ & $300-450$ & C \\
\hline \multirow[t]{2}{*}{106} & Blue-bearded Bee-eater & Nyctyornis athertoni & $\mathrm{R}(\mathrm{AM})$ & $750-900$ & UC \\
\hline & Monarchidae & & & & \\
\hline 107 & Asian Paradise-Flycatcher & Terpsiphone paradisi & $\mathrm{R}$ & $300-900$ & $\mathrm{C}$ \\
\hline \multirow[t]{2}{*}{108} & Black-naped Monarch-Flycatcher & Hypothymis azurea & $\mathrm{R}$ & $600-900$ & $\mathrm{C}$ \\
\hline & Motacillidae & & & & \\
\hline 109 & Paddy field Pipit & Anthus rufulus & $\mathrm{R}$ & $300-900$ & C \\
\hline 110 & Forest Wagtail & Dendronanthus indicus & M & $320-900$ & C \\
\hline 111 & Yellow Wagtail & Motacilla flava & M & $300-600$ & C \\
\hline 112 & Grey Wagtail & Moticella cinerea & M & $500-900$ & UC \\
\hline \multirow[t]{2}{*}{113} & Large Pied Wagtail & $\begin{array}{l}\text { Moticella } \\
\text { maderaspatensis }\end{array}$ & $\mathrm{R}$ & $300-900$ & UC \\
\hline & Muscicapidae & & & & \\
\hline 114 & Asian Brown Flycatcher & Muscicapa dauurica & M & $450-700$ & C \\
\hline 115 & Brown-breasted Flycatcher & Muscicapa muttui & M & $500-900$ & UC \\
\hline 116 & Blue-throated Flycatcher & Cyornis rubeculoides & $\mathrm{R}(\mathrm{AM})$ & 650 & $\mathrm{R}$ \\
\hline 117 & Tickell's Blue-Flycatcher & Cyornis tickelliae & $\mathrm{R}$ & $300-900$ & C \\
\hline 118 & White-rumped Shama & Copsycus malabaricus & $\mathrm{R}$ & $700-900$ & $\mathrm{C}$ \\
\hline 119 & Pied Bushchat & Saxicola caprata & $\mathrm{R}$ & $300-900$ & $\mathrm{C}$ \\
\hline 120 & Indian Robin & Saxicoloides fulicata & $\mathrm{R}$ & $300-640$ & C \\
\hline 121 & Blue-headed Rock-Thrush & Monticola cinclorhynchus & $M$ & 680 & $\mathrm{R}$ \\
\hline 122 & Indian Blue Robin & Luscinia brunnea & $\mathrm{R}$ & $300-900$ & C \\
\hline \multirow[t]{2}{*}{123} & Oriental Magpie-Robin & Copsycus saularis & $\mathrm{R}$ & $300-900$ & C \\
\hline & Nectariniidae & & & & \\
\hline 124 & Purple-rumped Sunbird & Nectarinia zeylonica & $\mathrm{R}$ & $300-900$ & $\mathrm{C}$ \\
\hline 125 & Small Sunbird & Nectarinia minima & $\mathrm{R}, \mathrm{E}$ & 900 & $\mathrm{R}$ \\
\hline 126 & Loten's Sunbird & Nectarinia Iotenia & $\mathrm{R}$ & $500-900$ & UC \\
\hline 127 & Purple Sunbird & Nectarinia asiatica & $\mathrm{R}$ & $300-900$ & $\mathrm{C}$ \\
\hline \multirow[t]{2}{*}{128} & Little Spiderhunter & Arachnothera longirostra & $\mathrm{R}$ & $650-900$ & $\mathrm{R}$ \\
\hline & Oriolidae & & & & \\
\hline 129 & Eurasian Golden Oriole & Oriolus oriolus & M & $300-350$ & C \\
\hline 130 & Black-naped Oriole & Oriolus chinensis & $M$ & $640-900$ & $\mathrm{C}$ \\
\hline \multirow[t]{2}{*}{131} & Black-headed Oriole & Oriolus xanthornus & $\mathrm{R}$ & $370-900$ & $\mathrm{C}$ \\
\hline & Paridae & & & & \\
\hline 132 & Great Tit & Parus major & $\mathrm{R}$ & $600-900$ & C \\
\hline \multirow[t]{2}{*}{133} & Black-lored Yellow Tit & Parus xanthogenys & $\mathrm{R}$ & $700-900$ & UC \\
\hline & Paroidea & & & & \\
\hline \multirow[t]{2}{*}{134} & Grey-headed Flycatcher & Culicicapa ceylonensis & $\mathrm{R}$ & $800-900$ & UC \\
\hline & Passeridae & & & & \\
\hline \multirow[t]{2}{*}{135} & House Sparrow & Passer domesticus & $\mathrm{R}$ & $300-900$ & C \\
\hline & Phalacrocoracidae & & & & \\
\hline 136 & Little Cormorant & Phalacrocorax niger & $\mathrm{R}$ & $300-900$ & C \\
\hline
\end{tabular}




\begin{tabular}{|c|c|c|c|c|c|}
\hline & Family/Species common name & Scientific name & Status & $\begin{array}{l}\text { Altitude } \\
\text { (in m) }\end{array}$ & $\begin{array}{c}\text { Frequency } \\
\text { of observation }\end{array}$ \\
\hline \multirow[t]{2}{*}{137} & Darter & Anhinga melanogaster & $\mathrm{R}, \mathrm{NT}$ & $300-900$ & $\mathrm{C}$ \\
\hline & Phasianidae & & & & \\
\hline 138 & Grey Francolin & $\begin{array}{l}\text { Francolinus } \\
\text { pondicerianus }\end{array}$ & $\mathrm{R}$ & $300-400$ & $\mathrm{C}$ \\
\hline 139 & Rain Quail & Coturnix coromandelica & $\mathrm{R}$ & $300-900$ & $\mathrm{C}$ \\
\hline 140 & Painted Bush-Quail & Perdicula erythrorhyncha & $\mathrm{R}$ & $300-900$ & $\mathrm{C}$ \\
\hline 141 & Red Spurfowl & Galloperdix spadicea & $\mathrm{R}$ & $500-900$ & $\mathrm{C}$ \\
\hline 142 & Painted Spurfowl & Galloperdix lunulata & $\mathrm{R}$ & $320-650$ & C \\
\hline 143 & Grey Junglefowl & Gallus sonneratii & $\mathrm{R}$ & $300-900$ & $\mathrm{C}$ \\
\hline \multirow[t]{2}{*}{144} & Indian Peafowl & Pavo cristatus & $\mathrm{R}$ & $300-500$ & C \\
\hline & Phylloscopidae & & & & \\
\hline 145 & Large-billed Leaf-Warbler & $\begin{array}{l}\text { Phylloscopus } \\
\text { magnirostris }\end{array}$ & M & $500-900$ & UC \\
\hline \multirow[t]{2}{*}{146} & Greenish Leaf-Warbler & Phylloscopus trochiloides & M & $320-900$ & $\mathrm{C}$ \\
\hline & Picidae & & & & \\
\hline 147 & Rufous Woodpecker & Celeus brachyurus & $\mathrm{R}$ & $600-640$ & $\mathrm{R}$ \\
\hline 148 & $\begin{array}{l}\text { Little Scaly-bellied Green } \\
\text { Woodpecker }\end{array}$ & Picus xanthopygaeus & $\mathrm{R}$ & $350-900$ & UC \\
\hline 149 & Small Yellow-naped Woodpecker & Picus chlorolophus & $\mathrm{R}$ & 665 & $\mathrm{C}$ \\
\hline 150 & $\begin{array}{l}\text { Lesser Golden-backed } \\
\text { Woodpecker }\end{array}$ & Dinopium benghalense & $\mathrm{R}$ & $300-900$ & $\mathrm{C}$ \\
\hline 151 & $\begin{array}{l}\text { Common Golden-backed } \\
\text { Woodpecker }\end{array}$ & Dinopium javanense & $\mathrm{R}$ & $300-900$ & $\mathrm{C}$ \\
\hline 152 & Yellow-fronted Pied Woodpecker & $\begin{array}{l}\text { Dendrocopos } \\
\text { mahrattensis }\end{array}$ & $\mathrm{R}$ & $642-900$ & UC \\
\hline 153 & $\begin{array}{l}\text { Brown-capped Pygmy } \\
\text { Woodpecker }\end{array}$ & Dendrocopos nanus & $\mathrm{R}$ & $780-900$ & UC \\
\hline \multirow[t]{2}{*}{154} & $\begin{array}{l}\text { Greater Golden-backed } \\
\text { Woodpecker }\end{array}$ & Chrysocolaptes Iucidus & $\mathrm{R}$ & $700-900$ & C \\
\hline & Pittidae & & & & \\
\hline \multirow[t]{2}{*}{155} & Indian Pitta & Pitta brachyura & M & $300-900$ & $\mathrm{C}$ \\
\hline & Ploceidae & & & & \\
\hline \multirow[t]{2}{*}{156} & Baya Weaver & Ploceus philippinus & $\mathrm{R}$ & $300-350$ & $\mathrm{C}$ \\
\hline & Podicipedidae & & & & \\
\hline \multirow[t]{2}{*}{157} & Little Grebe & Tachybaptus ruficollis & $\mathrm{R}$ & $300-900$ & $\mathrm{C}$ \\
\hline & Prionopidae & & & & \\
\hline 158 & Large Woodshrike & Tephrodornis gularis & $\mathrm{R}$ & $550-900$ & $\mathrm{C}$ \\
\hline 159 & Common Woodshrike & $\begin{array}{l}\text { Tephrodornis } \\
\text { pondicerianus }\end{array}$ & $\mathrm{R}$ & $320-700$ & C \\
\hline \multirow[t]{2}{*}{160} & Large Cuckoo-Shrike & Coracina macei & $\mathrm{R}$ & $550-700$ & $\mathrm{C}$ \\
\hline & Psittacidae & & & & \\
\hline 161 & Rose-ringed Parakeet & Psittacula krameri & $\mathrm{R}$ & $300-450$ & $\mathrm{C}$ \\
\hline 162 & Plum-headed Parakeet & Psittacula cyanocephala & $\mathrm{R}$ & $400-900$ & $\mathrm{C}$ \\
\hline 163 & Blue-winged Parakeet & Psittacula colomboides & $\mathrm{R}, \mathrm{E}$ & $500-900$ & C \\
\hline \multirow[t]{2}{*}{164} & Indian Hanging-Parrot & Loriculus vernalis & $\mathrm{R}$ & $500-900$ & $\mathrm{C}$ \\
\hline & Pycnonotidae & & & & \\
\hline 165 & Grey-headed Bulbul & Pycnonotus priocephalus & $\mathrm{R}, \mathrm{E}$ & $700-900$ & $\mathrm{C}$ \\
\hline 166 & Ruby-throated Yellow Bulbul* & $\begin{array}{l}\text { Pycnonotus melanicterus } \\
\text { gularis }\end{array}$ & $\mathrm{R}$ & $700-900$ & UC \\
\hline 167 & Red-whiskered Bulbul & Pycnonotus jocosus & $\mathrm{R}$ & $320-900$ & $\mathrm{C}$ \\
\hline
\end{tabular}




\begin{tabular}{|c|c|c|c|c|c|}
\hline & Family/Species common name & Scientific name & Status & $\begin{array}{l}\text { Altitude } \\
\text { (in } \mathrm{m} \text { ) }\end{array}$ & $\begin{array}{c}\text { Frequency } \\
\text { of observation }\end{array}$ \\
\hline 168 & Red-vented Bulbul & Pycnonotus cafer & $\mathrm{R}$ & $300-900$ & $\mathrm{C}$ \\
\hline 169 & Yellow-throated Bulbul & $\begin{array}{l}\text { Pycnonotus } \\
\text { xantholaemus }\end{array}$ & $\mathrm{R}, \mathrm{T}$ & $450-715$ & $\mathrm{R}$ \\
\hline 170 & White-browed Bulbul & Pycnonotus luteolus & $\mathrm{R}$ & $300-700$ & C \\
\hline \multirow[t]{2}{*}{171} & Yellow-browed Bulbul & Iole indica & $\mathrm{R}$ & $700-900$ & UC \\
\hline & Rallidae & & & & \\
\hline \multirow[t]{2}{*}{172} & White-breasted Waterhen & Amaurornis phoenicurus & $\mathrm{R}$ & $300-900$ & $\mathrm{C}$ \\
\hline & Rhipiduridae & & & & \\
\hline \multirow[t]{2}{*}{173} & White-browed Fantail-Flycatcher & Rhipidura aureola & $\mathrm{R}$ & 640 & C \\
\hline & Scolopacidae & & & & \\
\hline \multirow[t]{2}{*}{174} & Common Sandpiper & Actitis hypoleucos & M & $300-900$ & C \\
\hline & Sittidae & & & & \\
\hline \multirow[t]{2}{*}{175} & Velvet-fronted Nuthatch & Sitta frontalis & $\mathrm{R}$ & $700-900$ & UC \\
\hline & Strigidae & & & & \\
\hline 176 & Eurasian Eagle-Owl & Bubo bubo & $\mathrm{R}$ & 350 & $\mathrm{R}$ \\
\hline \multirow[t]{2}{*}{177} & Spotted Owlet & Athene brama & $\mathrm{R}$ & $300-900$ & C \\
\hline & Sturnidae & & & & \\
\hline 178 & Rosy Starling & Sturnus roseus & M & $320-400$ & UC \\
\hline 179 & Common Myna & Acridotheres tristis & $\mathrm{R}$ & $300-500$ & C \\
\hline 180 & Jungle Myna & Acridotheres fuscus & $\mathrm{R}$ & $450-900$ & UC \\
\hline \multirow[t]{2}{*}{181} & Southern Hill-Myna & Gracula indica & $\mathrm{R}$ & $750-900$ & $\mathrm{C}$ \\
\hline & Sylviidae & & & & \\
\hline \multirow[t]{2}{*}{182} & Yellow-eyed Babbler & Chrysomma sinense & $\mathrm{R}$ & $300-500$ & C \\
\hline & Timaliidae & & & & \\
\hline 183 & Indian Scimitar-Babbler & Pomatorhinus horsfieldii & $\mathrm{R}$ & $500-900$ & $\mathrm{C}$ \\
\hline 184 & Rufous-bellied Babbler & Dumetia hyperythra & $\mathrm{R}$ & $300-900$ & C \\
\hline 185 & Black-headed Babbler & Rhopocichla atriceps & $\mathrm{R}$ & $700-900$ & UC \\
\hline 186 & Common Babbler & Turdoides caudatus & $\mathrm{R}$ & $320-400$ & $\mathrm{C}$ \\
\hline 187 & Indian Rufous Babbler & Turdoides subrufus & $\mathrm{R}, \mathrm{E}$ & $540-900$ & $\mathrm{R}$ \\
\hline 188 & Jungle Babbler & Turdoides striatus & $\mathrm{R}$ & $450-900$ & C \\
\hline 189 & White-headed Babbler & Turdoides affinis & $\mathrm{R}$ & $300-400$ & $\mathrm{C}$ \\
\hline \multirow[t]{2}{*}{190} & Quaker Tit-Babbler & Alcippe poioicephala & $\mathrm{R}$ & $450-900$ & $\mathrm{R}$ \\
\hline & Trogonidae & & & & \\
\hline \multirow[t]{2}{*}{191} & Malabar Trogon & Harpactes fasciatus & $\mathrm{R}$ & $740-900$ & C \\
\hline & Turdidae & & & & \\
\hline \multirow[t]{2}{*}{192} & Malabar Whistiling-Thrush & Myiophonus horsfieldii & $\mathrm{R}$ & $500-900$ & C \\
\hline & Turnicidae & & & & \\
\hline 193 & Common Buttonquail & Turnix suscitator & $\mathrm{R}$ & $300-320$ & C \\
\hline \multirow[t]{2}{*}{194} & Small Buttonquail & Turnix sylvatica & $\mathrm{R}$ & 320 & C \\
\hline & Upupidae & & & & \\
\hline \multirow[t]{2}{*}{195} & Common Hoopoe & Upupa epops & $\mathrm{R}$ & $300-900$ & C \\
\hline & Zosteropidae & & & & \\
\hline 196 & Oriental White-eye & Zosterops palpebrosus & $\mathrm{R}$ & $550-900$ & $\mathrm{C}$ \\
\hline
\end{tabular}

R - Resident; C - Common; T- Threatened; M - Migrant; UC - Uncommon; NT - Near Threatened; AM - Altitudinal Migrant; X - Rare; E - Endemic 
additional eggs on 8 March. On 25 March the hatched eggshells were seen under the tree but the hatchlings were taken away by graziers for consumption. The nest was noticed again with a single egg on 5 April 2005, which was predated later. The other nest was located in early March on Acacia catechu near the arboretum of PHCC. It had two eggs and both fledged successfully on $2^{\text {nd }}$ May 2005.

Changeable Hawk-eagle Spizaetus cirrhatus: During the transect count on 24 March 2005 a nest was located containing a newly hatched chick with fluffy white down feathers and black beak on a tree located in a ravine of the riparian forest at an altitude of $732 \mathrm{~m}$. The nestling, being a singleton, was monitored continuously until fully grown and fledged. The nest was a large platform of sticks and twigs with a central depression lined with fresh green leaves constantly renovated to protect the nestling from direct sunlight. The nest was located at a height of about $15 \mathrm{~m}$ from the ground on a $30 \mathrm{~m}$ tall tree having a dbh (diameter at breast height) of $132 \mathrm{~cm}$.

Painted Spurfowl Galloperdix lunulata: A male with a single chick with dark brown down feathers was seen foraging on a rocky slope interspersed with grass and bush, near the road at $410 \mathrm{~m}$ altitude on 14 June 2005. It was speculated that both parents were involved in parental care.

Grey Junglefowl Gallus sonneratii: Noticed with three chicks on 25 March 2005, at $781 \mathrm{~m}$ altitude.

Indian Peafowl Pavo cristatus: Three juveniles were recorded on 11 December 2004 at $350 \mathrm{~m}$ altitude and may have possibly hatched in early November.

Little Brown Dove Streptopelia senegalensis: Three nests were observed in the Lower Palnis. At $320 \mathrm{~m}$ altitude the nest was made up of short grasses laid on the ground under Dodonaea viscosa bush. Another nest was located on an Acacia planifrons tree on 07 April 2005. A pair of eggshells from another nest was collected near Carissa sp. shrub on 14 June 2005.

Spotted Dove Streptopelia chinensis: On 2 July 2005 , a nest with two eggs was located on the lower fork of a Teak Tectona grandis tree at about $3 \mathrm{~m}$ from the ground at $320 \mathrm{~m}$ altitude.

Plum-headed Parakeet Psittacula cyanocephala: Two juveniles with an adult were seen at $600 \mathrm{~m}$ altitude on 7 April 2005.

Sirkeer Malkoha Phaenicophaeus leschenaultii:
A juvenile was seen perched on a Diospyros montana tree at $350 \mathrm{~m}$ altitude in mid April and another juvenile accompanying an adult was noticed in early June 2005.

Greater Coucal Centropus sinensis: This species was seen carrying food material in mid June at $320 \mathrm{~m}$ altitude.

Spotted Owlet Athene brama: The nest was seen on a rooftop of a house at $300 \mathrm{~m}$ altitude. Two broods were noticed in the same nest, one during February and another in late April. Both the broods were observed with eggs, but on the next day, all eggs rolled out of the nest to the ground.

Common Indian Nightjar Caprimulgus asiaticus: Two juveniles accompanying an adult were seen along the mud path at $320 \mathrm{~m}$ altitude on 5 May 2005. A juvenile was caught in a mist net in early December.

White-breasted Kingfisher Halcyon smyrnensis: A nest was located on the roadside bank at $630 \mathrm{~m}$ altitude, during May 2005.

Blue-bearded Bee-eater Nyctyornis athertoni: On 29 April 2005, a nest was located along the roadside along a gentle downward slope at a height of $850 \mathrm{~m}$ altitude. The nest was about $3-4 \mathrm{~cm}$ in diameter and more than $1.5 \mathrm{~m}$ deep.

Small Bee-eater Merops orientalis: On 24 April 2005 , at $320 \mathrm{~m}$ altitude a nest was observed in a small earthen structure constructed for rainwater harvesting. The nest hole was about two inches in diameter and both adults were seen hunting insects and feeding their nestlings. Many insect heads were seen scattered at the entrance of the nest.

Chestnut-headed Bee-eater Merops leschenaulti: In Lower Palnis, more than five nests were seen on a roadside sand bank on 17 April 2005 at $650 \mathrm{~m}$ altitude. These nests were horizontal tunnels drilled into the sand bank. An active nest was located and two eggshells were seen near the nest hole.

Malabar Grey Hornbill Ocyceros griseus: Two nests were noticed during March 2005 at an altitude of c. $800 \mathrm{~m}$. A nest-hole was found on a horizontal branch of a large Buchanania axillaris tree at a height of about $20 \mathrm{~m}$ from the ground and the other was on the trunk of a large tree with 10 nesting holes. In both the nests, the adult male was seen feeding the incarcerated inmates with black berries and insects. On 23 June 2005, two fledglings flew across the road.

Red-rumped Swallow Hirundo daurica: In the 
lower Palnis, three nests were located. Of them, two were constructed under road culverts, one at $415 \mathrm{~m}$ altitude and the other at $552 \mathrm{~m}$ altitude. The third nest was seen on the ceiling of an abandoned building at $320 \mathrm{~m}$ with shells of two eggs found on the ground. Only a single nest was seen with two nestlings.

Large cuckoo-shrike Coracina macei: A nest was noticed in the lower Palnis during early May 2005 at $604 \mathrm{~m}$ altitude. The nest was seen on the fork of a Tectona grandis tree at a height of about $20 \mathrm{~m}$. The nest was built by peeling the fibres from a Commiphora caudata tree. The nest was not found after the rains.

Small Minivet Pericrocotus cinnamomeus: Although the nest was not seen, both male and female were seen carrying food in mid April 2005 in the scrub forests at $320 \mathrm{~m}$ altitude.

Scarlet Minivet Pericrocotus flammeus: On 24 March 2005, a well-camouflaged nest lined with lichen was observed on Tectona grandis and the female was seen incubating the eggs. The male fed the incubating female a moth.

Black-crested Bulbul Pycnonotus melanicterus gularis: On 20 January 2005, a nest was located at $789 \mathrm{~m}$ altitude. The bird was seen plucking leaves for nest construction and taking them onto a tree.

Red-whiskered Bulbul Pycnonotus jocosus: Although no nests were located, two juveniles were caught in a mist net, at an altitude of $320 \mathrm{~m}$ on $1^{\text {st }}$ November 2004. As this species is very rare in the lower altitudes (up to $400 \mathrm{~m}$ ), the juveniles caught might be undergoing a 'juvenile dispersal period'.

Yellow-throated Bulbul Pycnonotus xantholaemus: Collection of nest materials by this bird was noticed on 10 April 2005 and 9 June 2005.

White-browed Bulbul Pycnonotus luteolus: A nest was located on 18 April 2005 on Celtis sp. tree at about $4 \mathrm{~m}$ from the ground at $630 \mathrm{~m}$ altitude.

Oriental Magpie-robin Copsychus saularis: On 7 May 2005, a bird was observed in the thick undergrowth with an insect in its mouth at $591 \mathrm{~m}$ altitude.

White-rumped Shama Copsychus malabaricus: A nest was located on a short dead stump of a tree on an inaccessible slope at $410 \mathrm{~m}$ altitude. Both the birds were seen frequenting the site as if they were feeding young ones.

Indian Robin Saxicoloides fulicata: The bird was seen carrying food to an inaccessible bush. Such sightings were made at various altitudes from $320-600$ $m$ altitude from mid May till late June.

Rufous-bellied Babbler Dumetia hyperythra: A nest with four nestlings was located at about $350 \mathrm{~m}$ on 30 April 2005 and all the nestlings fledged on 2 May 2005.

Yellow-eyed Babbler Chrysomma sinense: A nest with two nestlings was located on 17 November 2004.

White-headed Babbler Turdoides affinis: A nest with three eggs was located on a fork of Carissa sp. bush at about $1.5 \mathrm{~m}$ from the ground on 19 February 2005 at $320 \mathrm{~m}$ altitude. The eggs were predated and the nest was abandoned.

Jungle Prinia Prinia sylvatica: Three nests were located in the scrub jungle between altitudes 300 and $400 \mathrm{~m}$ from late February to May. Out of the three nests, two had nestlings (two and three) and the other nests with three eggs were abandoned later. The breeding season does not correspond with the months (April to September) as indicated by Ali \& Ripley (1983).

Plain Prinia Prinia inornata: A nest with two nestlings was located during the first week of December 2004 at $320 \mathrm{~m}$ altitude. Both the chicks fledged successfully.

Common Tailorbird Orthotomus sutorius: A total of 23 juveniles were ringed from September to June at $320 \mathrm{~m}$ altitude.

Asian Brown Flycatcher Muscicapa dauurica: A juvenile was noticed in a mixed foraging flock at $665 \mathrm{~m}$ altitude on 7 June 2005. An adult was noticed feeding a juvenile at $640 \mathrm{~m}$ altitude on 17 June 2005 .

Tickell's Blue-flycatcher Cyornis tickelliae: In the Lower Palnis, two nests with nestlings each, were located between 564 and $648 \mathrm{~m}$ altitudes respectively. Both the nests were placed in rocky clefts along the roadside. The first nest was located in late April 2005 which was well camouflaged with fern leaves and placed at about $5 \mathrm{~m}$ from the ground and the next was located in late June at about $4 \mathrm{~m}$ from the ground.

Tickell's Flowerpecker Dicaeum erythrorynchos: A male and female were observed collecting nest materials on 22 and 29 January 2005 at $550 \mathrm{~m}$ altitude in the dry deciduous forests of Lower Palni Hills.

Thick-billed Flowerpecker Dicaeum agile: Two nests were observed. A nest with two nestlings was being fed by both adults on an Acacia planifrons tree at c. $3 \mathrm{~m}$ from the ground on 29 January 2005 at $420 \mathrm{~m}$ 
altitude. The other was located on an Albizzia lebbeck tree at c. $5 \mathrm{~m}$ from the ground on 12 February 2005 at $580 \mathrm{~m}$ altitude.

Purple-rumped Sunbird Nectarinia zeylonica: Four nests were recorded, one each in January and February and two in March. All nests were abandoned.

Purple Sunbird Nectarinia asiatica: Ten nests were seen with two eggs each in lower Palnis. Nestlings in four nests fledged successfully, two nests were abandoned and the other two nests could not be monitored. A nest was located with two eggs on 28 April at 320m altitude in Acacia leucophloea tree, but both the hatchlings succumbed to rain on 3 May 2005.

Loten's Sunbird Nectarinia lotenia: At $462 \mathrm{~m}$ altitude a nest was located on 11 April 2005 at c. $6 \mathrm{~m}$ from the ground on a tree. Both the adults were seen frequenting the nest.

Oriental White-eye Zosterops palpebrosus: In lower Palnis, construction of two nests was observed on 24 March at altitudes 738 and $744 \mathrm{~m}$. One was at about $3 \mathrm{~m}$ from the ground on a Celtis sp. tree but was later damaged by a vehicle passing over. The other was at a height of about $8 \mathrm{~m}$ from the ground on a tree.

White-rumped Munia Lonchura striata: Two nests were recorded on 2 December 2004. One was located with seven nestlings on a tree about $3 \mathrm{~m}$ from the ground. One had an egg but no eggs were found in the other. Both the nests were abandoned.

White-throated Munia Lonchura malabarica: A nest with three eggs was located on an Acacia planifrons tree in late November 2005.

Common Myna Acridotheres tristis: A nest was constructed on the roof of a building at $320 \mathrm{~m}$ altitude during mid June and the the incubating eggs were seen till early July 2005.

Southern Hill-myna Gracula religiosa indica: A nest was observed at $800 \mathrm{~m}$ altitude in Lower Palnis on Chukrasia tubularis tree at c. $5 \mathrm{~m}$ from the ground during late March 2005. Reuse of the nest by the pair was observed in March and the second brood was seen in July 2005.

Black-headed Oriole Oriolus xanthornus: Collection of nest material was seen in late April and a juvenile was observed being fed by its parents in mid June 2005 at $619 \mathrm{~m}$ altitude.

Black Drongo Dicrurus macrocercus: On 24 March 2005, a nest with two eggs was located on Acacia leucophloea tree and both the eggs hatched on 5 April 2005.

Greater Racket-tailed Drongo Dicrurus paradiseus: In the lower Palnis, a nest with an incubating female was seen during mid April at $762 \mathrm{~m}$ altitude on a tall tree c. $20 \mathrm{~m}$ from the ground.

Indian Treepie Dendrocitta vagabunda: A mating pair was sighted in mid June 2005 at 360m altitude. A juvenile was seen begging for food from an adult at $630 \mathrm{~m}$ altitude on 6 July 2005 .

Jungle Crow Corvus macrorhynchos: A nest was located during mid-June on Azadirachta indica tree at a height of $7 \mathrm{~m}$ from the ground.

\section{DISCUSSION}

Species richness generally decreases with increasing elevation (Begon et al. 1996). Bird distribution and abundance varies with habitat (Jayapal et al. 2007; Ramesh et al. 2011), climatic condition, food resource and evolutionary history of the area (Jayson 1994). The species richness recorded was lowest in the upper Palnis ( $\mathrm{n}=99)$ and mid Palnis $(\mathrm{n}=130)$ (Balachandran et al. 2005) in comparison to lower Palnis. This was probably because lower Palnis has more deciduous and scrub jungle which may support high food availability. Thus, in this heterogeneous matrix of habitat it is essential to maintain and conserve intact forests. In Kalakad-Mundanthurai Tiger Reserve, Raman et al. (2005) revealed that bird community composition significantly correlated with elevation and tree species composition of sites, indicating the influence of deterministic factors on bird community structure. Resource abundance and availability are described as the most important factor in determining the community structure (Recher \& Davis 2002). The bird families Accipitridae, Cuculidae, Muscicapidae, Picidae, Timaliidae, Phasianidae, Pycnonotidae, Ardeidae, Cisticolidae, Columbidae, Dicruridae, Estrildidae, Motacillidae and Nectariniidae are sufficiently abundant and thus considered to be characteristic of resource availability in this region (Balachandran et al. 2005). Endemic bird species were less in the lower Palnis than mid and upper Palnis. Most of the endemics were confined to the higher altitudes due to the presence of moist evergreen and high altitude montane forests and grasslands 
(Pramod et al. 1997; Vijayan \& Gokula 2006). The extensive deciduous and scrub forests were not used much by the endemics though the habitats are generally richer in bird species than humid evergreen and montane forests, when equal areas are compared (Daniels et al. 1991, 1992). Similarly, the common and resident birds are abundant in lower Palnis. More bird species and families were found between 600 and $900 \mathrm{~m}$ altitude. This is probably related to the presence of mixed vegetation types at these altitudes. By comparing the number of species present in lower Palnis with earlier studies by Balachandran et al. (2005), and Somasundaram \& Vijayan (2008) in mid and upper Palnis the species richness was higher in the lower altitude than the higher altitude. Similarly, most studies have shown a general pattern of decreasing species richness with increasing altitude believed to mirror the well-recognised latitudinal gradient in species richness (MacArthur 1972; Bachman et al. 2004, Raman et al. 2005).

Palni Hills is located in the southeastern Western Ghats and is well known for its high endemism (Rajmohana \& Radhakrishnan 2008). Though these hills are highly significant for the seven high altitude endemic species namely Nilgiri Wood-Pigeon, Grey-breasted Laughing Thrush, Black-and-Orange Flycatcher, Nilgiri Flycatcher, Broad-tailed GrassWarbler, White-bellied Shortwing and Nilgiri Pipit, they are found above $1500 \mathrm{~m}$ (Balachandran et al. 2005; Somasundaram \& Vijayan 2008) despite the lower Palnis having a threatened species, three nearly threatened and five endemic species. In total, 9 and 11 endemics, 3 and 2 Vulnerable and 5 and 4 Near Threatened birds were recorded in mid and upper Palnis, respectively. The Indian Rufous Babbler Turdoides subrufus and the Small Sunbird Nectarinia minima are the two endemics seen in all the three divisions of Palnis. It is evident that Palni hills is a significant site due to the high congregation of threatened and endemic bird species as referred by the Important Bird Area (IBA) (Islam \& Rahmani 2004). This site has been selected as an IBA site on the basis of the presence of globally threatened species with a significant percentage of restricted range species, and some biome-restricted species (Islam \& Rahmani 2004). The presence of endemic and threatened species indicates the conservation importance of the study site. All the endemic species observed have already been recorded in the hill ranges of Palni Hills (Fairbank 1877; Terry 1887; Nichols 1937) while some that appeared to be rare like the Indian Rufous Babbler, Blue-winged Parakeet Psittacula columboides and Malabar Grey Hornbill Ocyceros griseus in Lower Palnis may be due to habitat fragmentation and degradation. Habitat loss due to anthropogenic pressure is the greatest threat to most of the Indian birds (Rahmani 2008). Anthropogenic pressures like illegal encroachment into forest land, livestock-grazing, and harvesting of fuel wood with huge quantities of minor forest products must be put under control in this region (Balachandran et al. 2005). Interestingly, variations in the observation of breeding season compared to Ali \& Ripley (1983) was noticed in plain Prinia Prinia inornata, known to breed from March to July, was found breeding in the first week of December. Its nest was located with two nestlings. Both nestlings fledged successfully. Shikra Accipiter badius breeds from March to June but it also bred earlier in February. Spotted Owlet Athene brama breeds in November and March but the second brood was observed in April. Common Iora Aegithina tiphia usually breeds from January to August, but it was noticed breeding in November along with the observation of a nest with juveniles. Baya Weaver Ploceus philippinus usually breeds from June to September but it was seen breeding in October.

Our study illustrated useful breeding observations of birds in the Palni Hills. The protection of Important Bird Areas contributes not only to bird conservation but even the biodiversity of this highly endangered ecosystem as a whole (Islam \& Rahmani 2004). Since we documented the occurrence, altitudinal distribution and breeding observations, we felt that eight months were sufficient to bring out useful information on the aforementioned aspects. However, we agree that the short term field study was a limitation and definitely a long term study would bring out robust ornithological information on Palni Hills. This study generated baseline data on bird species occurrence along an altitudinal gradient in the lower Palni Hills. Large scale habitat changes occurring globally fulfil endless human needs, habitat destruction, fragmentation and degradation necessitates further assessment on the impact of anthropogenic changes on birds (Brash 1987; Whitten et al. 1987; Khan et al. 1993). To maintain the bird community structure, further habitat exploitation 
must be minimised by regulating human activities (Johnsingh \& Joshua 1994). The data gathered from this study serves to be important in developing future conservation and management programs, which will identify priority areas for long-term persistence of bird communities.

\section{REFERENCES}

Ali, S. (1949). Indian Hill Birds. Oxford University Press, Bombay, 188pp.

Ali, S. \& S.D. Ripley (1983). Handbook of The Birds of India and Pakistan-Vol. 6. Second Edition. Oxford University Press, New Delhi, 462pp.

Ananthasubramaniam, C.K. (1979). Crows in Kodaikanal. Newsletter for Birdwatchers 19(7): 11-12.

Bachman, S., W.J. Baker, N. Brummitt, J. Dransfield \& J. Moat (2004). Altitudinal gradients, area and tropical island diversity: an example from the palms of New Guinea. Ecography 27: 299-310.

Balachandran, S. (1992). BNHS Bird Ringing activities at Kodai hills. Newsletter for Birdwatchers 32(7-8): 12-13.

Balachandran, S. (ed.) (1998). Bird Migration studies in India Final Report (1980-1992). Phase I: Population Structure and Movement of Indian Avifauna (1980-1986) Final Report Phase II: Bird Migration (1987-1992). Final Report. Bombay Natural History Society, Mumbai, 150pp.

Balachandran, S., A.R. Rahmani, N. Ezhilarasi, S. Babu, J.P.P. Chakravarthy \& T. Ramesh (2005). Revaluation of bird community structure of Palni Hills with special reference to threatened and endemic species. Final Report. Bombay Natural History Society, Mumbai, 105pp.

Begon, M., J.L. Harper \& C.R. Townsend (1996). Ecology: individuals, populations, and communities. Blackwell Science, London, 945pp.

Blanford, W.T. (1867). On a new species of Callene from the Pulney Hills in southern India. Proceedings of the Zoological Society of London 1867: 832-834.

Brash, A.R. (1987). The history of avian extinction and forest convertion on Puerto Rico. Biological Conservation 39: 97-111.

Champion, H.G. \& S.K. Seth (1968). A Revised Survey of the Forest Types of India. Government of India Publication, New Delhi, 404pp.

Chettri, N., E. Sharma \& D.C. Deb (2001). Bird community structure along a trekking corridor of Sikkim Himalaya: a conservation perspective. Biological Conservation 102: $1-16$.

Daniels, R.J.R., M. Hegde, N.V. Joshi \& M. Gadgil (1991). Assigning conservation value: a case study from India. Conservation Biology 5(4): 464-475.

Daniels, R.J.R., N.V. Joshi \& M. Gadgil (1992). On the relationship between bird and woody plant species diversity in the Uttara Kannada District of south India. Proceedings of the National Academy of Sciences (USA) 89(12): 53115315.

Fairbank, S.B. (1877). A list of birds collected and observed on the Palani Hills. Stray Feathers 5: 387-410.

Foulkes, R. (1904). The Indian edible-nest Swiftlet (Collocalia fuciphaga) in the Pulney Hills. Journal of the Bombay Natural History Society 15: 727.

Fuller, N. (1958). Additions to the birds of the Palni Hills (south India). Journal of the Bombay Natural History Society 55(1): 159-160.

Islam, M.Z. \& A.R. Rahmani (2004). Important Bird Areas in India: Priority Sites for Conservation. Indian Bird Conservation Network: Bombay Natural History Society and BirdLife International (UK), xxvii+1133pp.

Jayapal, R., Q. Qureshi \& R. Chellam (2007). Developing a spatial conservation protocol for Central Indian Highlands through a biogeographical analysis of birds and existing Protected Area network: A Geographical Information Systems approach. Research Report, Wildlife Institute of India, Dehradun, 200pp.

Jayson, E.A. (1994). Synecology and behavioural studies on the forest birds of Kerala. PhD Thesis, University of Calicut, Calicut, 314pp.

Johnsingh, A.J.T. \& J. Joshua (1994). Avifauna in three vegetation types on Mundanthurai Plateau, south India. Journal of Tropical Ecology 10: 323-335.

Kattan, G.H. \& P. Franco (2004). Bird diversity along elevational gradients in the Andes of Colombia: area and mass effects. Global Ecology and Biogeography 13: 451458.

Khan, J.A., D.N. Khan \& A. Ahmed (1993). Preliminary investigations of bird community structure at Aligarh, India. Tropical Ecology 34: 217-225.

Kremen, C. (1992). Assessing the indicator properties of the species assemblages for natural areas monitoring. Ecological Applications 2: 203-217.

Lee, P.-F., T.-S. Ding, F.-H. Hsu \& S. Geng (2004). Breeding bird species richness in Taiwan: distribution on gradients of elevation, primary productivity and urbanization. Journal of Biogeography 31: 307-314.

MacArthur, R.H. (1972). Geographical Ecology: Patterns in the Distribution of Species. Harper and Row, New York, 269pp.

Narayana, T.V. (1979). Birding in and around Kodaikanal. (ca. May 15-June 15). Newsletter for Birdwatchers 19(10): 2-5.

Navarro, A. (1955). Some new bird records in the Palni Hills, south India. Journal of the Bombay Natural History Society 53(1): 133-134.

Nichols, E.G. (1937). The Kodaikanal birds and how to name them. Journal of the Bombay Natural History Society 39(4): 812-830.

Pascal, J.P. (1988). Wet Evergreen Forests of the Western Ghats of India: Ecology, Structure, Floristic Composition and Succession. Institut Français de Pondichéry, India, 365pp. 
Pramod, P., N.V. Joshi, U. Ghate \& M. Gadgil (1997). On the hospitality of Western Ghats habitats for bird communities. Current Science 73(2): 122-127.

Prater, S.H. (1971). The Book of Indian Animals. Bombay Natural History Society, Oxford University Press, Bombay.

Rajmohana, K. \& C. Radhakrishnan (2008). Western Ghats- A Hotspot of Biological Wealth. Technical Report, Western Ghats Field Research Station, Zoological Survey of India, Kozhikode, Kerala, 32pp.

Rahmani, A.R. (2008). Flight to extinction. Spectrum, The Tribune. Retrieved June 8, 2008, from http://www.tribuneindia.com/2008/20080608/spectrum/Main1.htm.

Raman, T.R.S., N.V. Joshi \& R. Sukumar (2005). Tropical rainforest bird community structure in relation to altitude, tree species composition, and null models in the Western Ghats, India. Centre for Ecological Sciences, Indian Institute of Science, $34 \mathrm{pp}$.

Ramesh, T., N. Sridharan \& R. Kalle (2011). Birds of Kuno Wildlife Sanctuary, Central India. Zoo's Print 26(12): 25-29.

Recher, H.F. \& W.E. Davis (2002). Foraging profile of a Salmon Gum woodland avifauna in Western Australia. Journal of Royal Society of Western Australia 85: $103-111$.

Robin, V.V. \& R. Sukumar (2002). Status and habitat preference of White-bellied Shortwing Brachypteryx major in the Western Ghats (Kerala and Tamil Nadu), India. Bird Conservation International 12: 335-351.

Shahabuddin, G. (1997). Preliminary observations on the role of coffee plantations as avifaunal refuges in the Palni Hills of the Western Ghats. Journal of the Bombay Natural History Society 94(1): 10-21.

Shafiq, T., S. Javed \& J.A. Khan (1997). Bird community structure of middle altitude oak forest in Kumaon Himalayas, India: a preliminary investigation. International Journal of Ecology and Environmental Sciences 23: 389-400.

Somasundaram, S. \& L. Vijayan (2008). Foraging behaviour and guild structure of birds in the montane wet temperate forest of the Palni Hills, south India. Podoces 3(1/2): 79-91.

Steele, A. (1990). Birds of Petuparai, Kodaikanal. Newsletter for Birdwatchers 30 (7-8): 9.

Surendran, K.K. (1973). The crow in Kodaikanal, Palni Hills. India. Newsletter for Birdwatchers 13(8): 10.

Terry, H.A. (1887). A few additional notes on birds of the Pulney Hills. Stray Feathers 10: 467-480.

Vijayan L. \& V. Gokula (2006). Human Impact on the Bird Communities in the Western Ghats. In: Proceeding of the Chinese Acad. Sciences. Proceeding of the $23^{\text {rd }}$ International Ornithological Congress, Beijing 2002. Symposium paper. Acta Zoologica Sinica 52: 692-696.
Author Details: Dr. Tharmalingam Ramesh is broadly interested in mammal and bird ecology. He has conducted research on migratory birds, water birds, mist-netting, bird-ringing and birds of tropical forests in India. Presently he is a research fellow at the Wildlife Institute of India, studying predator-prey ecology in the Western Ghats. MR. J.P.P. CHAKRAVARTHY is a $\mathrm{Sr}$. Project Officer in the Western Ghats Landscape Program at WWF, studying the population of tiger, since 2010. DR. S. BALACHANDRAN is a Sr. Scientist at BNHS, studying migratory water birds and their movements across India. Ms. RIDDHIKA KALLE is pursuing her PhD at the Wildlife Institute of India (WII), on small carnivore ecology in Mudumalai Tiger Reserve. She worked as a research fellow at WII on predator-prey ecology in the Western Ghats.

Author Contribution: TR wrote the manuscript, designed the study, conducted field surveys, collected and analysed the data, JPPC and SB, designed the study, conducted field surveys and collected data, RK helped with the writing and editing of the manuscript. 